\title{
Funções de covariância para produção de leite no dia do controle em bovinos Gir leiteiro
}

\author{
Rodrigo Junqueira Pereira(1), Paulo Sávio Lopes ${ }^{(2)}$, Rui da Silva Verneque ${ }^{(3)}$, Mário Luiz Santana Júnior ${ }^{(4)}$, \\ Marcos Rodrigues Lagrotta(2) ${ }^{(2)}$ Robledo de Almeida Torres ${ }^{(2)}$
}

\begin{abstract}
(1)Universidade Estadual Paulista, Departamento de Zootecnia, Faculdade de Ciências Agrárias e Veterinárias, Rodovia Carlos Tonnani, Km 5, CEP 14870-000 Jaboticabal, SP. E-mail: rodjunper@yahoo.com.br (2)Universidade Federal de Viçosa, Departamento de Zootecnia, Avenida Peter Henry Rolfs, s/nº, Campus Universitário, CEP 36570-000 Viçosa, MG. E-mail: plopes@ufv.br, margrotta@yahoo.com.br, rtorres@ufv.br (3)Embrapa Gado de Leite, Rua Eugênio do Nascimento, no 610, Bairro Dom Bosco, CEP $36038-330$ Juiz de Fora, MG. E-mail: chpd@cnpgl.embrapa.br (4)Universidade de São Paulo, Departamento de Ciências Básicas, Faculdade de Zootecnia e Engenharia de Alimentos, Grupo de Melhoramento Animal e Biotecnologia, Avenida Duque de Caxias Norte, no 225, Campus da USP, CEP 13635-900 Pirassununga, SP. E-mail: 10mario@gmail.com
\end{abstract}

Resumo - O objetivo deste trabalho foi estimar funções de covariância para a produção de leite no dia do controle (PLDC). Foram analisados 27 mil registros de PLDC de 3.362 primeiras lactações de vacas da raça Gir leiteira, paridas entre 1990 e 2007. As PLDC foram agrupadas em vinte classes quinzenais, analisadas por modelos de regressão aleatória, cujos efeitos aleatórios, genético-aditivo e de ambiente permanente foram modelados pelas funções de Wilmink (W) ou Ali \& Schaeffer (AS). A modelagem da variância residual (VR) foi ajustada por meio de $1,4,6$ ou 10 classes. As estimativas de herdabilidade para a PLDC variaram de 0,12 a 0,32 , para a função AS, e de 0,09 a 0,33 , para a função W, e foram maiores ao início da lactação. As correlações genéticas entre as PLDC decresceram de valores próximos à unidade, entre controles adjacentes, para valores negativos entre as PLDC da primeira e duas últimas quinzenas da lactação. O modelo que empregou a função AS com quatro classes de VR é uma opção parcimoniosa para o ajuste das PLDC de vacas Gir leiteira no Brasil.

Termos para indexação: bovinos de leite, função paramétrica, inferência bayesiana, parâmetros genéticos.

\section{Covariance functions for test-day milk yield in Gir cattle}

\begin{abstract}
The objective of this work was to estimate covariance functions for test-day milk yield (TDMY). Twenty-seven thousand TDMY records of 3,362 first lactations of Gir cows calving between 1990 and 2007 were analyzed. The TDMY data were grouped in 20 biweekly classes, analyzed by random regression models, whose random, additive-genetic and permanent environment effects were fitted by Wilmink's (W) or Ali \& Schaeffer's (AS) functions. The residual variance (VR) modelling was fitted by 1, 4, 6, or 10 classes. The heritability estimates for TDMY varied from 0.12 to 0.32 for the AS function, and from 0.09 to 0.33 for W function, and were larger in the begining of the lactation period. The genetic correlations decreased from near unity, among adjacent TDMY values, to negative values between the first and the two last quarters of lactation. The model using the AS function with four VR classes, is a parsimonious option for the TDMY fitting of Gir cows in Brazil.
\end{abstract}

Index terms: dairy cattle, parametric function, Bayesian inference, genetic parameters.

\section{Introdução}

O controle leiteiro, geralmente realizado a intervalos de 30 dias, é uma prova zootécnica que consiste na mensuração e registro da produção individual da vaca em um período de 24 horas. Os registros do controle leiteiro constituem-se na única fonte de informação das avaliações genéticas de bovinos para a produção de leite. Tradicionalmente, tais registros são utilizados para a estimação da produção acumulada até 305 dias, medida esta utilizada nas avaliações genéticas.

Novas metodologias de avaliação genética vêm sendo estudadas e aplicadas nos países com programas consolidados de melhoramento genético de bovinos de leite e, entre essas, os modelos de regressão aleatória (MRA) (Henderson Junior, 1982) têm recebido grande destaque. Esses modelos utilizam registros de produção de leite no dia do controle (PLDC), em detrimento da produção acumulada até 305 dias de lactação.

Entre as diversas vantagens atribuídas ao uso dos MRA, destacam-se: a maximização da utilização dos registros de PLDC por animal, com potencial aumento da acurácia na avaliação dos indivíduos (Olori et al., 1999); a maior precisão na definição dos grupos contemporâneos e a quantificação dos efeitos ambientais a eles associados (Visscher \& Goddard, 1995); e a possibilidade de cálculo da persistência da produção de leite como as funções dos valores genéticos 
preditos para os dias da lactação. Adicionalmente, os MRA possibilitam a avaliação genética de animais cuja lactação ainda está em curso, sem a necessidade de projeções, o que viabiliza a realização de avaliações mais frequentes, permitindo uma redução no intervalo de geração (Swalve, 2000).

De acordo com Van Der Werf \& Schaeffer (1997), uma função de covariância (FC) pode ser definida como uma função contínua que fornece as covariâncias de características medidas em diferentes pontos de uma trajetória (lactação), ao descrever as covariâncias entre as medidas tomadas em determinadas idades (dias em lactação) como função dessas idades. Meyer \& Hill (1997) demonstraram que os coeficientes da FC podem ser estimados a partir dos MRA. Assim, diferentes funções podem ser empregadas para ajustar a trajetória ao longo do tempo. Entre as funções paramétricas, destacam-se a função exponencial de Wilmink (Wilmink, 1987) e a função logarítmica de Ali \& Schaeffer (Ali \& Schaeffer, 1987).

Alguns autores têm proposto modificações dessas funções para buscar um melhor ajuste aos dados (Brotherstone et al., 2000; Jakobsen et al., 2002; Freitas, 2003). Uma das modificações mais estudadas é a alteração do valor do parâmetro a3, da função exponencial de Wilmink, que está relacionado ao período em que ocorre o pico de produção na lactação e também ao formato do pico. Tradicionalmente, é considerado constante e igual a 0,05 . Alterações no valor desse parâmetro podem melhorar o ajuste da função para uma população, raça ou espécie. Para a raça Gir leiteira, não foram localizados estudos que contemplem esses aspectos.

Segundo El Faro \& Albuquerque (2003), desconsiderar a heterogeneidade de variância residual nos estudos que utilizam MRA pode acarretar partição incorreta da variação total. Assim, a utilização de variância residual constante dentro de - mas heterogênea entre - classes de período de lactação tem sido estudada (El Faro \& Albuquerque, 2003; Costa et al., 2005; Melo et al., 2007; Costa et al., 2008; Herrera et al., 2008).

Há apenas dois estudos na literatura (Costa et al., 2005; Herrera et al., 2008) sobre funções de covariância para a produção de leite no dia do controle na raça Gir leiteira, nenhum dos quais utilizou o banco de dados do Arquivo Zootécnico Nacional, o único que contém informações de toda a população da raça.
Portanto, outros estudos são necessários para que se possa viabilizar a utilização dos MRA nas avaliações genéticas nacionais da raça Gir leiteira.

O objetivo deste trabalho foi estimar funções de covariância quanto à produção de leite no dia do controle de primeiras lactações de vacas Gir leiteira, pelas funções paramétricas exponencial (de Wilmink) e logarítmica (de Ali \& Schaeffer), por meio de modelos de regressão aleatória.

\section{Material e Métodos}

Foram utilizados 62.758 registros de produção de leite no dia do controle (PLDC) relativos a 7.552 primeiras lactações de vacas Gir leiteira, paridas entre 1990 e 2007, com idade ao parto entre 24 e 60 meses. Os dados são originários do Arquivo Zootécnico Nacional de Gado de Leite, sob gerenciamento da Embrapa Gado de Leite. Foram utilizados os controles entre o $5^{\circ}$ e o 305 dias da lactação. Foram estabelecidas as seguintes condições para a inclusão das vacas no estudo: primeiro controle leiteiro realizado antes de 45 dias após o parto; intervalo de controles leiteiros entre 15 e 45 dias; número de controles leiteiros superior a três. Em adição, condicionou-se a disponibilidade de grupo contemporâneo, caracterizado por rebanho-ano-mês de controle (RAM) com no mínimo três vacas, filhas de pelo menos dois touros. As PLDC foram agrupadas em vinte classes quinzenais de dias em lactação (Tabela 1), e foram eliminadas da análise as vacas com produções superiores ou inferiores a três desvios-padrão da média da quinzena da lactação. Após essas medições, foram utilizados nas análises 27 mil registros de PLDC de 3.362 vacas, filhas de 507 touros, em 56 rebanhos localizados principalmente na região Sudeste, mas também nas regiões Nordeste, Centro-Oeste e Sul. Após buscar o parentesco entre os animais por cinco gerações, totalizaram-se 8.590 animais no arquivo de pedigree.

Duas formas de estrutura de variância residual foram avaliadas para cada modelo: variância residual constante ao longo da lactação ou variância residual constante dentro de - mas heterogênea entre - classes de dias em lactação. Quando heterogêneas, foram avaliadas quatro, seis ou dez classes de variâncias residuais. Nos modelos com dez classes, as quinzenas foram agrupadas como: $1^{\mathrm{a}}-2^{\mathrm{a}}, 3^{\mathrm{a}}-4^{\mathrm{a}}, 5^{\mathrm{a}}-6^{\mathrm{a}}, 7^{\mathrm{a}}-8^{\mathrm{a}}, 9^{\mathrm{a}}-10^{\mathrm{a}}$, $11^{\mathrm{a}}-12^{\mathrm{a}}, 13^{\mathrm{a}}-14^{\mathrm{a}}, 15^{\mathrm{a}}-16^{\mathrm{a}}, 17^{\mathrm{a}}-18^{\mathrm{a}}$ e $19^{\mathrm{a}}-20^{\mathrm{a}}$. Quando 
foram empregadas quatro ou seis classes, as variâncias foram agrupadas por semelhança, com base nos modelos com dez classes de variância. Nos modelos com quatro e seis classes, as quinzenas foram agrupadas como:

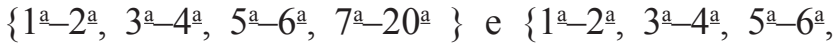
7 $\left.-8^{\mathrm{a}}, 9^{\mathrm{a}}-18^{\mathrm{a}}, 19^{\mathrm{a}}-20^{\mathrm{a}}\right\}$, respectivamente.

Nas regressões fixa, genético-aditiva e de ambiente permanente, foram utilizadas as funções paramétricas que se seguem. A função logarítmica de Ali \& Schaeffer (Ali \& Schaeffer, 1987) (AS), que é caracterizada por cinco parâmetros: $\mathrm{y}=\mathrm{a} 0+\mathrm{a} 1 \mathrm{c}+\mathrm{a} 2 \mathrm{c} 2+\mathrm{a} 3(\ln 1 / \mathrm{c})+$ a4 $(\ln 1 / \mathrm{c}) 2$, em que: $\mathrm{c}=\mathrm{t} / 305$; $\mathrm{t}$ representa os dias em lactação; e y é a produção de leite no dia do controle. A função exponencial de Wilmink (Wilmink, 1987) (W), é caracterizada por quatro parâmetros: $\mathrm{y}=\mathrm{a} 0$ $+\mathrm{a} 1 \mathrm{t}+\mathrm{a} 2 \exp (-\mathrm{a} 3 \mathrm{t})$, em que: $\mathrm{t}$ representa os dias em lactação e y é a produção de leite no dia do controle. $\mathrm{Na}$ função $\mathrm{W}$, o parâmetro a3 está relacionado ao momento da lactação em que ocorre o pico e ao formato do mesmo.

Para a função de Wilmink, foram avaliados diferentes valores para o parâmetro a3: 0,06, 0,05 (padrão), 0,04, $0,03,0,025,0,02,0,015$ e 0,01 . Os modelos ajustados com esta função foram identificados por W seguido de um valor que representa o parâmetro a3 $(06,05,04,03$, 025, 02, 015 ou 01).
O modelo de regressão aleatória geral, utilizado nas análises, é representado por:

$\mathrm{y}_{\mathrm{ijk}}=\mathrm{RAM}_{\mathrm{i}}+\sum_{\mathrm{n}=1}^{2} \mathrm{~b}_{\mathrm{n}} \mathrm{x}_{\mathrm{j}}^{\mathrm{n}}+\sum_{\mathrm{m}=0}^{\mathrm{o}-1} \beta_{\mathrm{m}} \varphi_{\mathrm{m}}(\mathrm{t})+\sum_{\mathrm{m}=0}^{\mathrm{o}-1} \alpha_{\mathrm{jm}} \varphi_{\mathrm{m}}(\mathrm{t})+\sum_{\mathrm{m}=0}^{\mathrm{o}-1} \mathrm{p}_{\mathrm{jm}} \varphi_{\mathrm{m}}(\mathrm{t})+\varepsilon_{\mathrm{ij} k}$,

em que: $y_{i j k}$ é a k-ésima observação, registrada no dia de lactação $t$ do animal $j$, no rebanho-ano-mês de controle (RAM) i; RAM é o efeito do i-ésimo grupo contemporâneo (2.325 classes); $b_{n}$ é o coeficiente de regressão para os efeitos linear $(n=1)$ e quadrático $(n=2)$ da PLDC, em função da idade $x_{j}$ da vaca ao parto, em meses; $\beta_{\mathrm{m}}$ é o conjunto de $\mathrm{m}$ coeficientes de regressão fixos para a trajetória média da população; $\varphi_{\mathrm{m}}(\mathrm{t})$ é a covariável da função de regressão de ordem $\mathrm{o}$, de acordo com o dia da lactação $(\mathrm{t}) ; \alpha_{j m}, \mathrm{p}_{\mathrm{jm}}$ são os conjuntos de $\mathrm{m}$ coeficientes de regressão aleatórios genéticos aditivos e de ambiente permanentes, para cada vaca $\mathrm{j}$; e $\varepsilon_{\mathrm{ijk}}$ é o erro aleatório ou medida de erro temporário, associada à observação $k$ da vaca $j$ pertencente ao rebanho-ano-mês de controle i.

$\mathrm{O}$ modelo de regressão aleatória pode ser reescrito, na forma matricial por: $\mathrm{y}=\mathrm{X} \beta+\mathrm{Za}+\mathrm{Wp}+\varepsilon$, em que: y é o vetor das observações; $\beta$ é o vetor dos efeitos fixos; a é o vetor dos coeficientes de regressão aleatória do efeito genético aditivo de animal; p é o vetor dos coeficientes de regressão aleatória do

Tabela 1. Produção de leite de bovinos de raça Gir leiteira, entre 1990 e 2007, de acordo com a quinzena do controle e o dia da lactação $0^{(1)}$.

\begin{tabular}{|c|c|c|c|c|c|c|}
\hline Quinzena & Dias em lactação & Dia médio & $\mathrm{N}$ & Média (kg) & $\mathrm{DP}(\mathrm{kg})$ & CV (\%) \\
\hline 1 & $5-19$ & 12 & 1338 & 10,0 & 3,7 & 37,2 \\
\hline 2 & $20-34$ & 27 & 1616 & 10,7 & 3,7 & 35,0 \\
\hline 3 & $35-49$ & 42 & 1596 & 10,9 & 3,9 & 36,1 \\
\hline 4 & $50-64$ & 57 & 1570 & 10,4 & 3,6 & 34,8 \\
\hline 5 & $65-79$ & 72 & 1601 & 10,3 & 3,7 & 36,3 \\
\hline 6 & $80-94$ & 87 & 1539 & 10,0 & 3,5 & 35,4 \\
\hline 7 & $95-109$ & 102 & 1581 & 9,7 & 3,6 & 37,0 \\
\hline 8 & $110-124$ & 117 & 1469 & 9,6 & 3,4 & 36,1 \\
\hline 9 & $125-139$ & 132 & 1481 & 9,4 & 3,5 & 36,9 \\
\hline 10 & $140-154$ & 147 & 1381 & 9,2 & 3,4 & 36,9 \\
\hline 11 & $155-169$ & 162 & 1416 & 9,0 & 3,3 & 36,7 \\
\hline 12 & $170-184$ & 177 & 1345 & 8,7 & 3,2 & 36,8 \\
\hline 13 & 185-199 & 192 & 1348 & 8,6 & 3,2 & 37,5 \\
\hline 14 & $200-214$ & 207 & 1272 & 8,3 & 3,1 & 37,2 \\
\hline 15 & $215-229$ & 222 & 1284 & 8,1 & 3,1 & 38,2 \\
\hline 16 & $230-244$ & 237 & 1160 & 7,8 & 3,1 & 39,4 \\
\hline 17 & $245-259$ & 252 & 1160 & 7,8 & 3,1 & 40,0 \\
\hline 18 & $260-274$ & 267 & 1032 & 7,5 & 3,0 & 39,6 \\
\hline 19 & $275-289$ & 282 & 973 & 7,4 & 3,0 & 40,8 \\
\hline 20 & $290-305$ & 297 & 838 & 7,3 & 2,9 & 39,8 \\
\hline
\end{tabular}

(1)N, número de observações; DP, desvio-padrão; e CV, coeficiente de variação. 
efeito de ambiente permanente; $\varepsilon$ é o vetor de efeito aleatório residual; e $\mathrm{X}, \mathrm{Z}$ e $\mathrm{W}$ são as matrizes de incidência, correspondentes às observações para efeitos fixos, efeitos aleatórios de animal e de ambiente permanente, respectivamente, para os quais se assume:

$$
\mathrm{y} \mid \mathrm{b}, \mathrm{a}, \mathrm{p}, \sigma_{\mathrm{e}_{1}}^{2}, \ldots, \sigma_{\mathrm{e}_{\mathrm{x}}}^{2} \sim \mathrm{NMV}(\mathrm{Xb}+\mathrm{Za}+\mathrm{Wp}, \mathrm{R}),
$$

que é a distribuição de y (observações), dados os parâmetros do modelo. $\mathrm{R}=\operatorname{diag}\left\{\sigma_{\mathrm{e}_{\mathrm{x}}}^{2}\right\}$, em que $\mathrm{x}$ varia de 1 até $\mathrm{n}$ e $\mathrm{n}$ é o número de classes de resíduo.

As pressuposições acerca das distribuições a priori, para os parâmetros desconhecidos, foram consideradas como a seguir (Rekaya et al., 1999): $\mathrm{a} \mid \Lambda_{\mathrm{a}} \sim \mathrm{NMV}(0, \mathrm{G})$, com $\mathrm{G}=\mathrm{A} \otimes \Lambda_{\mathrm{a}}$, em que: A é a matriz do numerador dos coeficientes de parentesco entre os animais; e $\Lambda_{\mathrm{a}}$ é a matriz que contém as covariâncias entre os coeficientes de regressão aleatória genéticos aditivos; $\mathrm{p} \mid \Lambda_{\mathrm{p}} \sim \mathrm{NMV}(0, \mathrm{P})$, com $\mathrm{P}=\mathrm{I} \otimes \Lambda_{\mathrm{p}}$, em que: I é uma matriz identidade e $\Lambda_{\mathrm{p}}$ é a matriz contendo as covariâncias entre os coeficientes de regressão aleatória de ambiente permanente;

$$
\Lambda_{\mathrm{a}}\left|v_{\mathrm{a}}, \mathrm{S}_{\mathrm{a}}^{2} \sim \mathrm{W}^{-1}\left(v_{\mathrm{a}}, v_{\mathrm{a}} \mathrm{S}_{\mathrm{a}}^{2}\right), \Lambda_{\mathrm{p}}\right| v_{\mathrm{p}}, \mathrm{S}_{\mathrm{p}}^{2} \sim \mathrm{W}^{-1}\left(v_{\mathrm{p}}, v_{\mathrm{p}} \mathrm{S}_{\mathrm{p}}^{2}\right) \mathrm{e}
$$

$\mathrm{R} \mid v_{\mathrm{e}}, \mathrm{S}_{\mathrm{e}}^{2} \sim \chi^{-2}\left(v_{\mathrm{e}}, v_{\mathrm{e}} \mathrm{S}_{\mathrm{e}}^{2}\right)$, em que $v_{\mathrm{a}}, \mathrm{S}_{\mathrm{a}}^{2}, v_{\mathrm{p}}, \mathrm{S}_{\mathrm{p}}^{2}$, e $v_{\mathrm{e}}, \mathrm{S}_{\mathrm{e}}^{2}$ representam o grau de confiança e os valores a priori para as covariâncias dos coeficientes de regressão genético-aditivos e de ambiente permanente, e para as variâncias residuais, respectivamente.

$\mathrm{NMV}, \mathrm{W}^{-1}$ e $\chi^{-2}$ referem-se às distribuições normal multivariada, Wishart invertida e qui-quadrado invertida escalonada, respectivamente. Detalhes sobre as distribuições condicionais completas a posteriori foram descritos por Jamrozik \& Schaeffer (1997) e Rekaya et al. (1999).

As inferências sobre os parâmetros de interesse foram realizadas a partir de suas correspondentes distribuições marginais posteriores, com o algoritmo "Gibbs sampling" (Geman \& Geman, 1984). Para cada modelo avaliado, foram geradas 550 mil amostras. Após o descarte das primeiras 50 mil amostras, procedeu-se a um intervalo de descarte amostral de 40, tendo restado 12.500 amostras, com base nas quais as inferências foram realizadas. Nas análises, utilizaram-se os programas escritos em linguagem Fortran90, GIBBS3F90 e POSTGIBBSF90 (Misztal, 2008).
Os modelos de regressão aleatória foram comparados pelo "deviance information criterion (DIC)", apresentado por Spiegelhalter et al. (2002):

$\mathrm{DIC}=\overline{\mathrm{D}}(\theta)+\mathrm{p}_{\mathrm{D}}=2 \overline{\mathrm{D}}(\theta)-\mathrm{D}(\theta)$, em que $: \overline{\mathrm{D}}(\theta)=\mathrm{E}_{\theta \mid \mathrm{y}}[\mathrm{D}(\theta)]$ (esperança posterior da "deviance" bayesiana) e $\mathrm{D}(\theta)=-2 \log \mathrm{p}(\mathrm{y} \mid \theta)$ representam a qualidade de ajuste do modelo. O número efetivo de parâmetros representa a penalização para o incremento da complexidade do modelo: $\mathrm{p}_{\mathrm{D}}=\overline{\mathrm{D}}(\theta)-\mathrm{D}(\bar{\theta})$, em que $\theta$ é o vetor de parâmetros do modelo, e $\mathrm{D}(\bar{\theta})$ é a "deviance" bayesiana avaliada às médias posteriores dos parâmetros. Modelos com menor DIC são preferidos por indicar melhor qualidade de ajuste, combinada com menor grau de complexidade.

\section{Resultados e Discussão}

A produção média de leite no dia do controle foi de 9,2 kg, com desvio-padrão de $3,6 \mathrm{~kg}$ e coeficiente de variação de $38,9 \%$. Houve aumento da produção nas primeiras três quinzenas (Tabela 1). A partir da terceira quinzena, a produção decresceu gradativamente até o final da lactação. Em relação ao número de controles leiteiros por vaca, $80 \%$ delas tinham entre sete e dez controles.

Para a função paramétrica exponencial de Wilmink (W), sob homogeneidade de variância residual, o melhor ajuste foi observado quando o valor do parâmetro a3 foi considerado 0,025, segundo os valores de DIC. Como este parâmetro tem relação com o dia da lactação em que ocorre o pico, pode-se inferir que o pico de produção da população em estudo ocorre mais cedo na lactação, em comparação ao da raça Holandesa, para a qual a função de Wilmink original (parâmetro a3 = $0,05)$ foi desenvolvida.

Emrelaçãoà estrutura de variância residual, os valores de DIC indicaram que os modelos que consideravam homogeneidade de variância residual promoveram o pior ajuste, para ambas as funções empregadas na modelagem das trajetórias média (regressão fixa), genética aditiva e de ambiente permanente (Tabela 2). Os resultados sugerem que a heterogeneidade de variâncias residuais deve ser considerada, uma vez que as variâncias mostraram comportamento diferenciado ao longo da lactação. Esses resultados estão de acordo com os de Costa et al. (2005) e Herrera et al. (2008), ambos realizados com a raça Gir leiteira.

Para a função AS, o modelo com quatro classes de variância residual (VR) foi o mais adequado, de acordo 
com o critério DIC, o qual indica melhor qualidade de ajuste combinada ao menor grau de complexidade (Tabela 2). Em relação à função W025, foi escolhido o modelo com seis classes de VR. Assim, para a função AS, o modelo com quatro classes de VR (AS-4) seria adequado para o ajuste aos dados, enquanto para a função W025, aquele com seis classes de VR (W025-6) seria recomendado.

Para a função AS, o modelo que considerava homogeneidade de VR (AS-1) a subestimou no terço inicial da lactação e a superestimou nos terços seguintes (Figura 1A). Para a função W, foi observado comportamento semelhante no modelo com homogeneidade de VR (W025-1). Entretanto, adicionalmente, a VR foi subestimada nas duas últimas quinzenas da lactação (Figura 1B). Quando comparados os modelos AS-4 e W025-6 no contexto das VR, o primeiro apresentou estimativas de VR inferiores, o que confirma seu melhor ajuste aos dados, e deve ser preferido para as avaliações genéticas quanto à produção de leite, nesta população, por modelos de regressão aleatória.

As estimativas médias posteriores das variâncias genéticas para os modelos AS-4 e W025-6 apresentaram tendências semelhantes, com valores que decresceram de 2,28-2,78 $\mathrm{kg}^{2}$ ao início da lactação até $0,59-0,61 \mathrm{~kg}^{2}$ ao final da lactação (Figura 2 A). O modelo W025-6 tendeu a superestimar a variância genética na primeira quinzena e a subestimá-la nas três últimas quinzenas da lactação. Em relação às variâncias de ambiente permanente, ambos os modelos proporcionaram estimativas semelhantes,

Tabela 2. Número de classes de variância residual (r), número de parâmetros (p) e "deviance information criterion (DIC)", para cada modelo de regressão aleatória sob diferentes estruturas residuais, de bovinos da raça Gir leiteira entre 1990 e 2007.

\begin{tabular}{cccc}
\hline Modelo & $\mathrm{r}$ & $\mathrm{p}$ & $\mathrm{DIC}$ \\
\hline W025 & 1 & 13 & 88.072 \\
1 & 4 & 16 & 88.013 \\
2 & 6 & 18 & 87.967 \\
3 & 10 & 22 & 88.068 \\
4 & & & \\
\hline AS & 1 & 31 & 85.344 \\
1 & 4 & 34 & $85.073^{(1)}$ \\
2 & 6 & 36 & 85.152 \\
3 & 10 & 40 & 85.177 \\
4
\end{tabular}

${ }^{(1)}$ Melhor modelo com base no DIC. com valores que oscilam entre 3,48 e $4,68 \mathrm{~kg}^{2}$ ao longo da lactação (Figura 2 B).

As herdabilidades médias posteriores para os modelos AS-4 e W025-6 tiveram comportamento semelhante no decorrer da lactação, exceto nos extremos, quando as diferenças entre os modelos foram encontradas (Figura 3). O modelo AS-4 proporcionou estimativas inferiores na primeira quinzena e superiores nas últimas quatro quinzenas da lactação, em comparação ao W025-6. Para AS-4, as estimativas médias de herdabilidade aumentaram até a segunda quinzena, atingiram o valor máximo de 0,32 e decresceram ao longo da lactação até a 17 a quinzena $(0,12)$, a partir da qual voltaram a crescer até 0,15 (última quinzena). Para W025-6, os valores estimados variaram de 0,33 (primeira quinzena) a 0,09 (última quinzena). As estimativas de herdabilidade apresentaram, como esperado, a mesma tendência dos componentes de variância dos efeitos aleatórios genéticos aditivos.
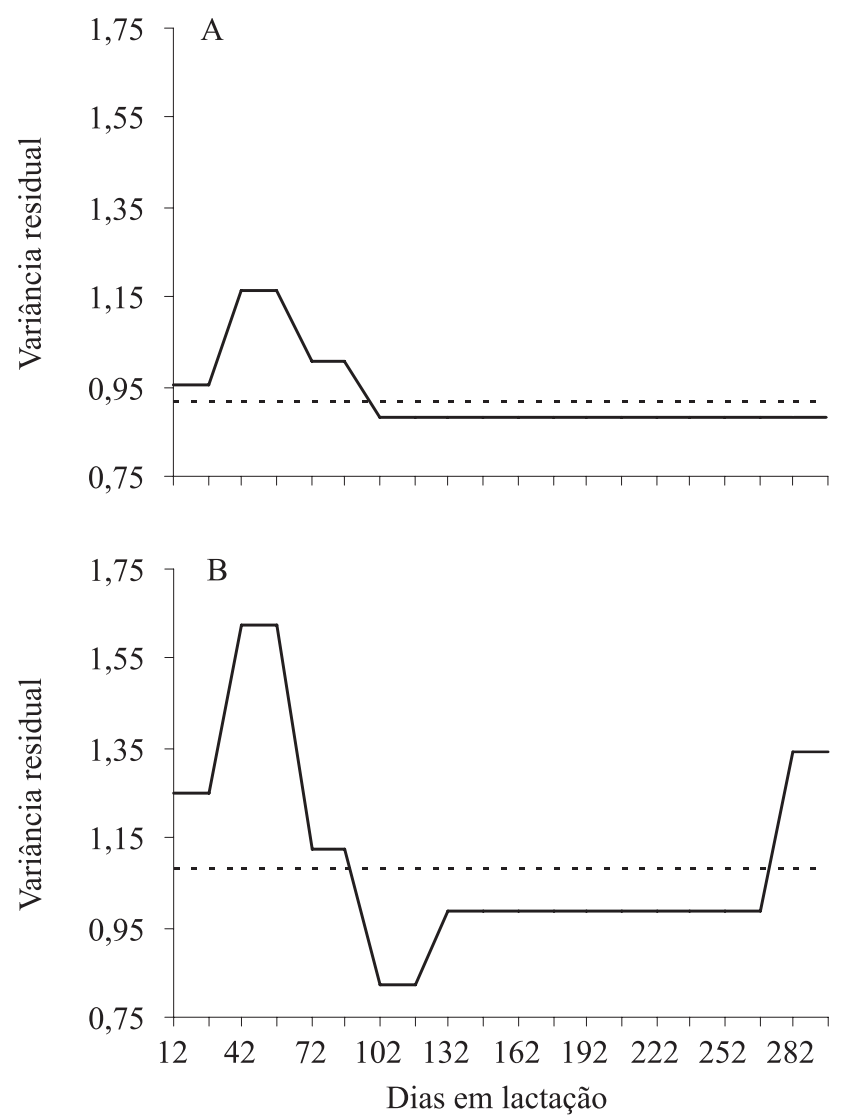

Figura 1. Médias posteriores de variância residual $\left(\mathrm{kg}^{2}\right)$, para a produção de leite de bovinos da raça Gir leiteira entre 1990 e 2007, para os modelos AS-1 (- - -) e AS-4 (-) (A), W025-1 (- - -) e W025-6 (-) (B). 
Herrera et al. (2008), trabalharam com dados da mesma raça e obtiveram estimativas semelhantes, tendo encontrado, no entanto, estimativas decrescentes do início $(0,33)$ ao fim $(0,21)$ da lactação, para um modelo que utilizou a função logarítmica de Ali \& Schaeffer.

As superfícies que representam as correlações genéticas, de ambiente permanente e fenotípicas indicam a mesma tendência para os modelos AS-4 e W025-6 (Figura 4). No contexto das correlações genéticas, observaram-se valores médios posteriores próximos à unidade entre as PLDC adjacentes e uma redução nos mesmos valores conforme o intervalo de dias entre as mensurações das PLDC. Estimativas negativas foram encontradas entre as PLDC dos extremos da lactação. Brotherstone et al. (2000), ao empregar as funções paramétricas AS e $\mathrm{W}$, para a raça Holandesa, observaram estimativas negativas entre o primeiro controle e os controles a partir dos 42 dias de lactação. Resultados semelhantes foram relatados por Rekaya et al. (1999), López-Romero \& Carabaño
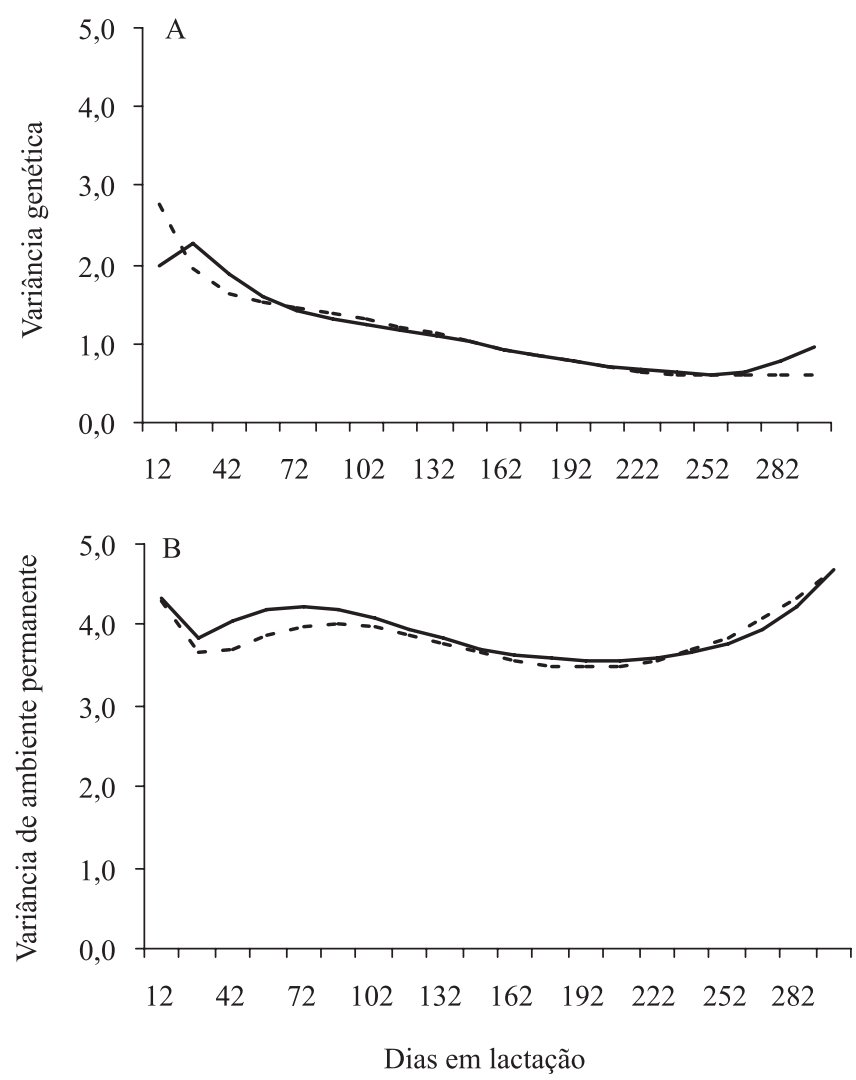

Figura 2. Estimativas médias posteriores de variâncias genética $\left(\mathrm{kg}^{2}\right)(\mathrm{A})$ e de ambiente permanente $\left(\mathrm{kg}^{2}\right)(\mathrm{B})$, para os modelos AS-4 (-) e W025-6 (- - ), ao longo da lactação de bovinos da raça Gir leiteira entre 1990 e 2007.
(2003) e Melo et al. (2007) para a raça Holandesa, por Kettunen et al. (2000) para a raça Ayrshire, e por Costa et al. (2005) para a raça Gir.

Meyer (1999) relatou que o ajuste dos modelos de regressão aleatória, nas extremidades da curva, é deficiente, provavelmente em razão do menor número de observações e da variação amostral na partição da variância total, o que pode levar à obtenção de estimativas erradas dos parâmetros genéticos. Outros fatores, de origem não genética, que possivelmente também interferem na partição da variância total da produção de leite são os efeitos da gestação e do uso de somatotropina, geralmente não incluídos nos modelos de avaliação genética de bovinos leiteiros.

Tem sido reportado o efeito negativo da gestação na produção de leite de vacas leiteiras, em parte como consequência do aumento dos requerimentos nutricionais do feto no decorrer da gestação, o que reduz a disponibilidade de nutrientes para a produção (Bell et al., 1995). Assim, vacas de menor potencial de produção poderiam ser pouco afetadas pela gestação, pois seus requerimentos nutricionais são pequenos. Em contrapartida, vacas de maior potencial produtivo sofreriam forte influência da gestação, em razão da alta competição nutricional com o feto, principalmente nos estádios finais da gestação-lactação. Tal fato geraria diferenças consideráveis entre as produções nos extremos da lactação e afetaria a partição da variância total, interferindo consequentemente nas estimativas dos parâmetros genéticos.

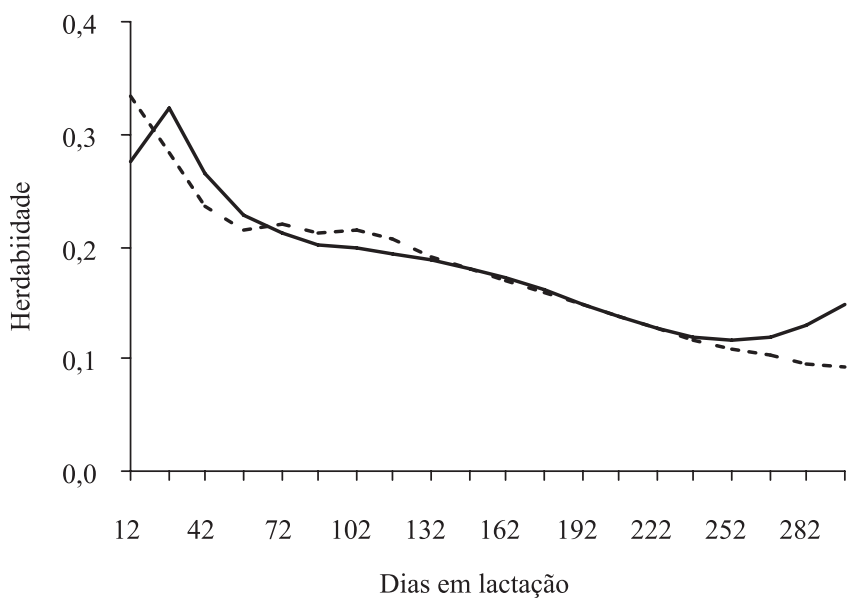

Figura 3. Estimativas médias posteriores de herdabilidade, para a produção de leite de bovinos da raça Gir leiteira entre 1990 e 2007, para os modelos AS-4 (-) e W025-6 (- - -), ao longo da lactação. 
A recomendação técnica para utilização da somatotropina (bST) é de que seja aplicada somente após o pico de lactação. Normalmente, em adição à sua aplicação, é dada alimentação mais concentrada. Assim, vacas que recebem esse tratamento podem ter produções ao final da lactação superiores às iniciais, o que pode interferir nas estimativas de parâmetros genéticos.

Os modelos de regressão aleatória são ideais em avaliações genéticas que consideram os efeitos não genéticos da gestação e do uso de bST, pois permitem quantificá-los no momento em que incidem na lactação.
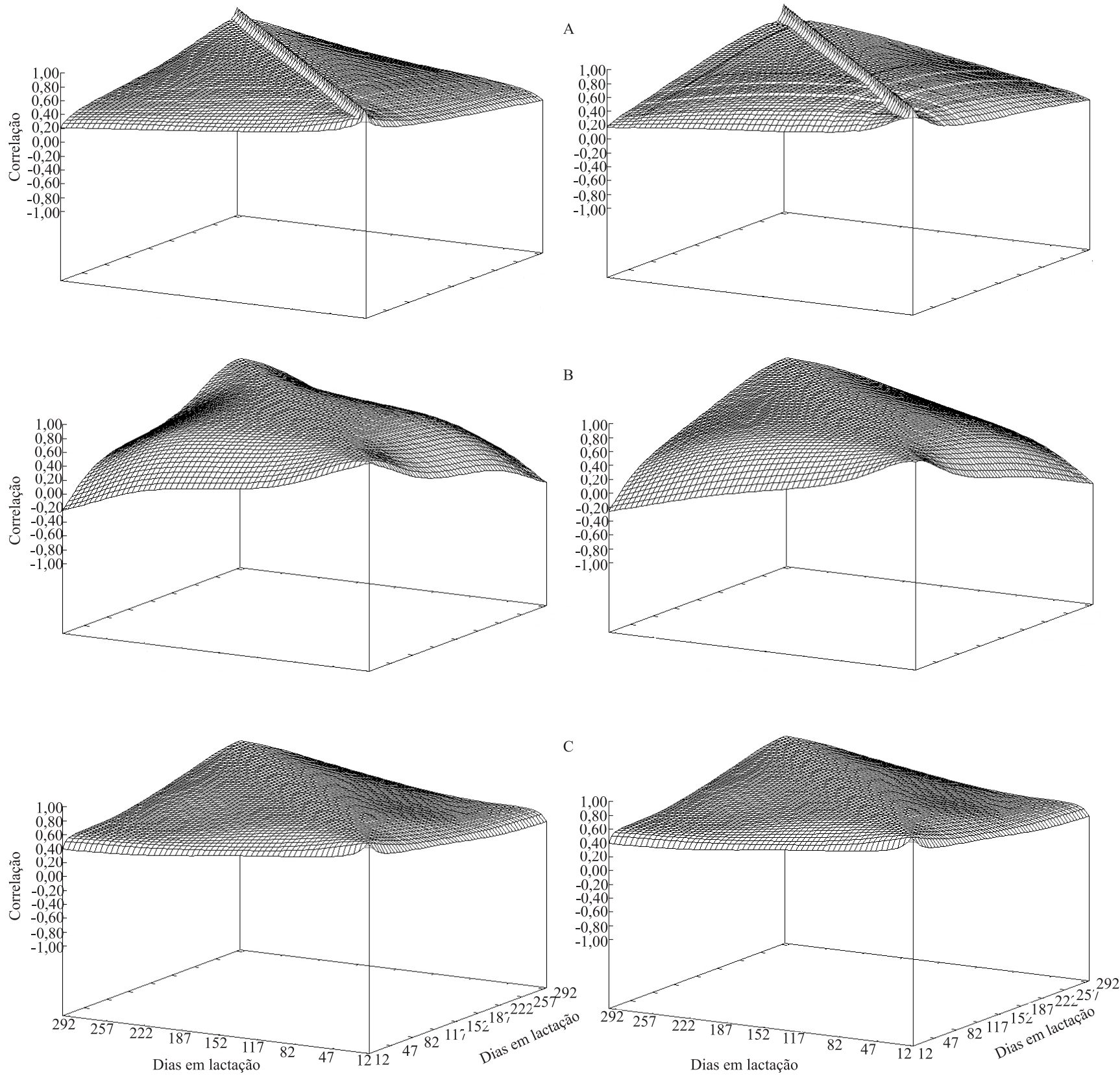

Figura 4. Estimativas médias posteriores de correlações fenotípicas (A), genéticas (B) e de ambiente permanente (C), entre as produções de leite de bovinos da raça Gir leiteira entre 1990 e 2007, obtidas com os modelos AS-4 (esquerda) e W025-6 (direita). 
Atualmente, dezoito países utilizam modelos de produção no dia do controle, em suas avaliações genéticas nacionais, dezesseis dos quais por meio de modelos de regressão aleatória (Interbull, 2009). Alguns desses países como Holanda, Canadá e Finlândia realizam o cálculo do valor genético para persistência da produção de leite, característica funcional relacionada à redução de custos com alimentação, saúde e reprodução.

A perspectiva de uso dos modelos de regressão aleatória, nas avaliações genéticas da raça Gir leiteira, estimula a identificação de outras funções para modelagem da produção de leite no dia do controle, que permitem um ajuste de melhor qualidade.

\section{Conclusões}

1. A heterogeneidade de variâncias residuais deve ser considerada no ajuste da produção de leite no dia do controle de vacas da raça Gir leiteira, por modelos de regressão aleatória, com uso das funções de Ali \& Schaeffer e de Wilmink, uma vez que as variâncias mostram comportamento diferenciado ao longo da lactação.

2. A função de Ali \& Schaeffer proporciona melhor qualidade de ajuste, em comparação à de Wilmink, e deve ser preferida nas avaliações genéticas da raça Gir leiteira.

3. O modelo que usa a função de Ali \& Schaeffer, com quatro classes de variância residual, para a modelagem da produção de leite no dia do controle de vacas da raça Gir leiteira, na primeira lactação, por modelos de regressão aleatória, caracteriza-se pela parcimônia e pode ser utilizado nas avaliações genéticas para produção de leite dessa raça.

\section{Agradecimentos}

À Coordenação de Aperfeiçoamento de Pessoal de Nível Superior, por concessão de bolsa; ao Conselho Nacional de Desenvolvimento Científico e Tecnológico e à Fundação de Amparo à Pesquisa de Minas Gerais, por suporte financeiro; à Embrapa Gado de Leite, pela concessão do acesso aos dados do Arquivo Zootécnico Nacional e pela cessão do espaço de trabalho que tornou possível este estudo.

\section{Referências}

ALI, T.E.; SCHAEFFER, R. Accounting for covariances among test day milk yields in dairy cows. Canadian Journal of Animal Science, v.67, p.637-644, 1987.

BELL, A.W.; SLEPETIS, R.; EHRHARDT, R.A. Growth and accretion of energy and protein in the gravid uterus during late pregnancy in Holstein cows. Journal of Dairy Science, v.78, p.1954-1961, 1995.

BROTHERSTONE, S.; WHITE, I.M.S.; MEYER, K. Genetic modelling of daily yield using orthogonal polynomials and parametric curves. Animal Science, v.70, p. 407-415, 2000.

COSTA, C.N.; MELO, C.M.R. de; MACHADO, C.H.C.; FREITAS, A.F. de; PACKER, I.U.; COBUCI, J. de A. Parâmetros genéticos para a produção de leite de controles individuais de vacas da raça Gir estimados com modelos de repetibilidade e regressão aleatória.

Revista Brasileira de Zootecnia, v.34, p.1519-1530, 2005.

COSTA, C.N.; MELO, C.M.R. de; PACKER, I.U.; FREITAS, A.F. de; TEIXEIRA, N.M.; COBUCI, J. de A. Genetic parameters for test day milk yield of first lactation Holstein cows estimated by random regression using Legendre polynomials. Revista Brasileira de Zootecnia, v.37, p.602-608, 2008.

EL FARO, L.; ALBUQUERQUE, L.G. de. Utilização de modelos de regressão aleatória para produção de leite no dia do controle, com diferentes estruturas de variâncias residuais. Revista Brasileira de Zootecnia, v.32, p.1104-1113, 2003.

FREITAS, M.S. Utilização de modelos de regressão aleatória na avaliação genética de animais da raça Girolando. 2003. 78p. Dissertação (Mestrado) - Universidade Federal de Viçosa, Viçosa.

GEMAN, S.; GEMAN, D. Stochastic relaxation, Gibbs distributions and the Bayesian restoration of images. IEEE Transactions on Pattern Analysis and Machine Intelligence, v.12, p.421-741, 1984.

HERRERA, L.G.G.; EL FARO, L.; ALBUQUERQUE, L.G. de; TONHATI, H.; MACHADO, C.H.C. Estimativas de parâmetros genéticos para a produção de leite e persistência da lactação em vacas Gir, aplicando modelos de regressão aleatória. Revista Brasileira de Zootecnia, v.37, p.1584-1594, 2008.

HENDERSON JUNIOR, C.R. Analysis of covariance in the mixed model: higher-level, nonhomogeneous, and random regressions. Biometrics, v.38, p.623-640, 1982.

INTERBULL. Genetic evaluations. 2009. Available at: $<$ http:// www-interbull.slu.se/eval/framesida-prod.htm>. Accessed on: 8 . Dec. 2010.

JAKOBSEN, J.H.; MADSEN, P.; JENSEN, J.; PEDERSEN, J.; CHRISTENSEN, L.G.; SORENSEN, D.A. Genetic parameters for milk production and persistency for Danish Holsteins estimated in random regression models using REML. Journal of Dairy Science, v.85, p.1607-1616, 2002.

JAMROZIK, J.; SCHAEFFER, L.R. Estimates of genetic parameters for a test day model with random regressions for yield traits of first lactation Holsteins. Journal of Dairy Science, v.80, p.762-770, 1997. 
KETTUNEN, A.; MÄNTYSAARI, E.A.; PÖSÖ, J. Estimation of genetic parameters for daily milk yield of primiparous Ayrshire cows by random regression test-day models. Livestock Production Science, v.66, p.251-261, 2000.

LÓPEZ-ROMERO, P.; CARABAÑO, M.J. Comparing alternative random regression models to analyse first lactation daily milk yield data in Holstein-Friesian cattle. Livestock Production Science, v.82, p.81-96, 2003.

MELO, C.M.R. de; PACKER, I.U.; COSTA, C.N.; MACHADO, P.F. Genetic parameters for test day milk yields of first lactation Holstein cows by random regression models. Animal, v.1, p.325-334, 2007.

MEYER, K. Estimates of genetic and phenotypic covariance functions for postweaning growth and mature weight of beef cows. Journal of Animal Breeding and Genetics, v.116, p.181-205, 1999.

MEYER, K.; HILL, W.G. Estimation of genetic and phenotypic covariance functions for longitudinal or "repeated" records by restricted maximum likelihood. Livestock Production Science, v.47, p.185-200, 1997.

MISZTAL, I. BLUPF90: a flexible mixed model program in Fortran 90. 2008. Available at: <http://nce.ads.uga.edu/ ignacy/ numpub/blupf90/docs/>. Accessed on: 08 Dec. 2010.
OLORI, V.E.; HILL, W.G.; MCGUIRK, B.J.; BROTHERSTONE, S. Estimating variance components for test day milk records by restricted maximum likelihood with a random regression animal model. Livestock Production Science, v.61, p.53-63, 1999.

REKAYA, R.; CARBAÑO, M.J.; TORO, M.A. Use of test day yields for the genetic evaluation of production traits in Holstein-Friesian cattle. Livestock Production Science, v.57, p.203-217, 1999.

SPIEGELHALTER, D.J.; BEST, N.G.; CARLIN, B.R.; VAN DER LINDE, A. Bayesian measures of model complexity and fit. Journal of the Royal Statistical Society: Series B: Statistical Methodology, v.64, p.583-639, 2002.

SWALVE, H.H. Theoretical basis and computational methods for different test-day genetic evaluation methods. Journal of Dairy Science, v.83, p.1115-1124, 2000.

VAN DER WERF, J.; SCHAEFFER, L. Random regression in animal breeding. Ontário: University of Guelf, 1997. 70p.

VISSCHER, P.M.; GODDARD, M.E. Genetic parameters for milk yield, survival, workability and type traits for Australian dairy cattle. Journal of Dairy Science, v.78, p.205-220, 1995.

WILMINK, J.B.M. Adjustment of test-day milk, fat and protein yields for age, season and stage of lactation. Livestock Production Science, v.16, p.335-348, 1987.

$\overline{\text { Recebido em } 28 \text { de agosto de } 2010 \text { e aprovado em } 8 \text { de outubro de } 2010}$ 